\title{
Retrorectus repair of incisional ventral hernia with urinary bladder matrix reinforcement in a long-term porcine model
}

\author{
D Adam Young ${ }^{1}$, Nicolette Jackson ${ }^{2}$, Catherine A Ronaghan ${ }^{3}$, Collin EM Brathwaite ${ }^{4}$ \& \\ Thomas W Gilbert*,1,5 \\ ${ }^{1}$ ACell Inc., Columbia, MD 21046, USA \\ ${ }^{2}$ Alizée Pathology LLC, Thurmont, MD 21788, USA \\ ${ }^{3}$ Department of Surgery, Texas Tech University, Lubbock, TX 79409, USA \\ ${ }^{4}$ Department of Surgery, NYU Winthrop Hospital, Mineola, NY 11501, USA \\ ${ }^{5}$ McGowan Institute for Regenerative Medicine, University of Pittsburgh, Pittsburgh, PA 15219, USA \\ *Author for correspondence: Tel.. +1 410953 8509; Fax: +1 410715 4511; thomasgilbert@acell.com
}

\begin{abstract}
Aim: Not all biologically derived materials elicit the same host response when used for reinforcement of ventral hernia repairs. This study aimed to evaluate the remodeling characteristics of the abdominal wall following reinforcement with urinary bladder matrix (UBM) in a large animal preclinical model of ventral hernia repair. Materials \& methods: Midline defects in 36 Yucatan minipigs were reinforced with UBM-derived surgical devices using a classic Rives-Stoppa-Wantz approach, and compared with primary repair controls. After 3 or 8 months, the abdominal wall was explanted for histological and mechanical analysis. Results \& conclusion: All UBM-derived surgical devices were completely resorbed within 8 months and facilitated deposition of vascularized, biomechanically functional connective tissue in the retrorectus plane, with no evidence of hernia formation.
\end{abstract}

First draft submitted: 5 March 2017; Accepted for publication: 20 April 2018; Published online: 4 May 2018

\section{Keywords: abdominal $\bullet$ ECM $\bullet$ hernia $\bullet$ posterior fascia $\bullet$ retrorectus}

The incidence of ventral hernia continues to rise annually in the USA and it is estimated that nearly $10 \%$ of all laparotomies result in incisional hernias [1,2]. Substantial clinical evidence has shown that primary suture repair of a ventral hernia alone results in unacceptably high recurrence rates $(>50 \%)$, and that all incisional hernia repairs should be reinforced with surgical mesh [3,4]. However, there are numerous surgical mesh materials available for hernia reinforcement, with limited consensus on the ideal material for this application. Given the high cost of hernia recurrence and increasing pressure to reduce procedural costs, it is critical to choose the appropriate surgical mesh material for primary reinforcement that effectively reduces the risk of postoperative complications while warranting the additional cost $[5,6]$.

For low-risk patients with limited comorbidities (Grade 1 patients in the Ventral Hernia Working Group classification), synthetic meshes, such as those derived from polypropylene and polyethylene, are typically favored due to the low initial cost and clinical history of successful use $[7,8]$. As the risk of contamination or other surgical site occurrences increases in clean-contaminated and contaminated cases, biologically derived materials, such as those processed from human or animal extracellular matrix (ECM), increase in use due to the susceptibility of synthetic meshes to biofilm formation and mesh erosion $[9,10]$. Biologically derived materials are intended to provide a natural scaffold for host tissue integration and revascularization, while also providing mechanical reinforcement to the repair. However, just as different synthetic polymers elicit different reactions in the body, subtle differences in the processing and composition of various biologically derived materials can result in different host responses that can have meaningful impacts on the clinical outcome [11,12].

For example, the use of detergents or crosslinking agents can significantly alter the host response to an ECM material [12]. A recent evaluation of the steps involved in producing a dermal ECM material revealed that each step resulted in substantial changes to the content, structure and mechanical behavior of the resulting ECM [13]. 
Accordingly, a prospective analysis of clinical biopsies of different dermal ECM materials utilized in abdominal wall repairs showed significant differences in neovascularization, cellular infiltration, tissue deposition and degradation based on the species of the ECM source (human, porcine or bovine) [14]. Similarly, a separate review of clinical biopsies from 14 explanted meshes also revealed increased encapsulation in crosslinked dermal ECM materials compared with noncrosslinked dermal ECM, as well as a lack of remodeling and revascularization in both groups [15]. In this study, while these materials were likely still providing mechanical support to the abdominal wall, other nonmechanical factors contributed to the mesh failure in these patients that possibly could have been avoided if the dermal ECM had integrated better with the surrounding tissue. This highlights a clinical need for a biologically derived material that can be utilized in complex abdominal wall repairs that not only provides mechanical reinforcement, but also facilitates remodeling and revascularization by the host at the site of device placement.

Urinary bladder matrix (UBM) is a form of porcine ECM that is derived from the inner lining of the urinary bladder. UBM is manufactured without the use of harsh detergents or other chemical agents and thus is able to maintain much of the structure and composition of the native ECM, as previously described $[16,17]$. Extensive literature has previously shown that UBM is rapidly remodeled in vivo and results in site-appropriate tissue deposition by the body in a variety of anatomies $[18,19]$. Preclinical evidence has reported that UBM is associated with favorable host responses such as immune cell modulation, neovascularization, re-innervation and progenitor cell participation [20-24]. Clinical case studies that utilized commercialized versions of MatriStem $U_{B M}{ }^{T M}$ for wound management (i.e., Cytal ${ }^{\circledR}$ Wound Matrix and MicroMatrix ${ }^{\circledR}$; ACell Inc., MD, USA) have also shown that UBM can support deposition of granulation tissue and re-epithelialization in a variety of challenging applications, such as complex traumatic wounds, contaminated wounds and wounds with exposed avascular structures [25-27]. Similarly, there is growing evidence for the successful clinical use of UBM in various surgical reinforcement applications [28,29].

Recently, a preclinical small animal model of an abdominal wall defect repaired with a multilaminate sheet of UBM showed that the UBM was rapidly remodeled by the host into a fibrous connective tissue that maintained mechanical strength at or above that of the native rat abdominal wall [30]. Interestingly, the strength of the remodeled tissue remained well after the UBM had completely been resorbed. Thus, the study described herein aimed to evaluate if similar remodeling characteristics and mechanical strength would also be seen in a more clinically relevant porcine preclinical model of ventral hernia repair. For this study, three different configurations of UBM-derived surgical devices were utilized, which differed only in the arrangement of the individual pieces of UBM within the device and the thickness of the multilaminate sheet. The differing arrangements of the UBM sheets allow for devices to be made with increasing surface area beyond the size constraints of an individual bladder. It was hypothesized that each of these UBM-derived surgical devices would limit hernia recurrence by providing initial mechanical support, and perhaps more importantly, by facilitating the deposition of host tissue that was capable of supporting the physiologic loading of the abdominal wall well after the device had fully resorbed.

\section{Materials \& methods}

\section{Study design}

This study utilized an acute midline abdominal defect model in adult female Yucatan minipigs $(30-50 \mathrm{~kg})$ with a classic Rives-Stoppa-Wantz retromuscular approach to simulate hernia repair. The study was conducted in compliance with Title 21 of the US FDA Code of Federal Regulations Part 58: Good Laboratory Practice. A total of 32 animals were randomly assigned to one of four treatment groups: Group 1: primary suture repair of the midline defect; Group 2: reinforcement of the primary repair with Gentrix ${ }^{\circledR}$ Surgical Matrix Plus (ACell Inc.); Group 3: reinforcement with Gentrix Surgical Matrix Thick Configuration \#1; or Group 4: reinforcement with Gentrix Surgical Matrix Thick Configuration \#2. Animals were survived for time points of 3 or 8 months, with four animals per treatment group per time point. An additional four animals were not subjected to surgery and utilized as a baseline for mechanical strength of the native abdominal wall. The study design and animal usage were reviewed and approved by the Institutional Animal Care and Use Committee for compliance with all applicable regulations and welfare laws and policies. All animal procedures and housing were in compliance with the USDA Animal Welfare Act, the Guide for the Care and Use of Laboratory Animals, the Guide for the Care and Use of Agricultural Animals in Research and Teaching, Public Health Service National Institutes of Health Office of Laboratory Animal Welfare and the American Veterinary Medical Association Guidelines for Euthanasia. All surgical procedures were conducted under the supervision of an independent study director and all data were audited by the respective Quality Assurance Unit. 

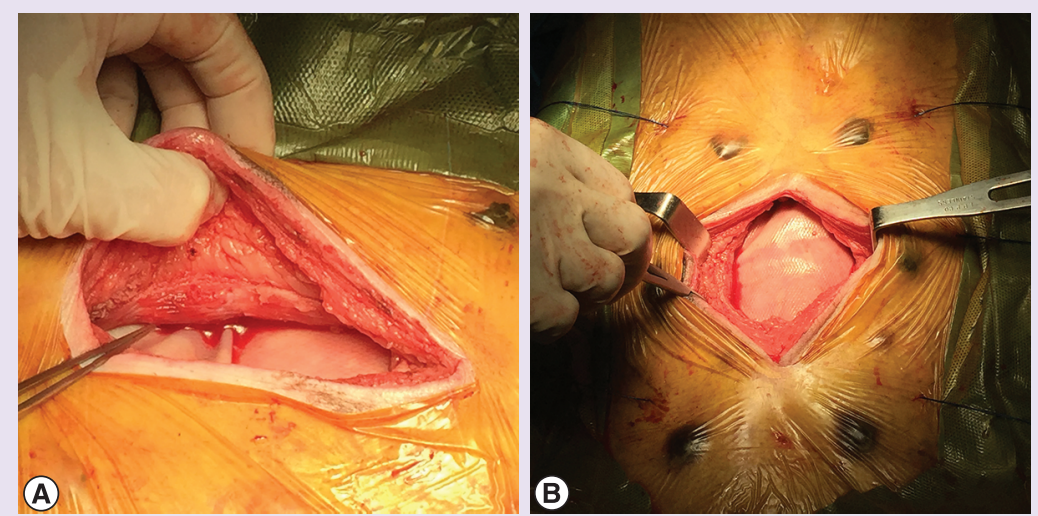

Figure 1. Surgical procedure. (A) This porcine model simulated a ventral hernia repair through creation of a midline defect and preparation of the retrorectus plane using the classic Rives-Stoppa-Wantz approach. The primary repair was reinforced with a UBM-derived surgical device placed between the rectus muscle and posterior fascia. (B) The UBM-derived surgical device was secured in the retrorectus plane with transfascial sutures. UBM: Urinary bladder matrix.

\section{Surgical procedure}

A single midline abdominal wall defect measuring $10 \mathrm{~cm}$ on the cranial-caudal axis, approximately $5 \mathrm{~cm}$ cranial and $5 \mathrm{~cm}$ caudal relative to the umbilicus, was made using electrocautery through the skin, subcutaneous fat, aponeurotic muscle and fascial layers of the abdominal wall. Care was taken to avoid violating the peritoneum. Following the Rives-Stoppa-Wantz retromuscular approach, blunt dissection was used to define the retromuscular plane on either side of the midline defect between the rectus muscle and the posterior fascia of the rectus sheath ('retrorectus'). The surgeon remained blinded to animal assignment until placement of a reinforcement device. Study materials were hydrated in sterile saline according to the instructions for use. The UBM-derived surgical device was placed such that the basement membrane side of the device faced the peritoneum. Each $10 \times 15 \mathrm{~cm}$ device was oriented with the long edge $(15 \mathrm{~cm})$ running axially and the short edge $(10 \mathrm{~cm})$ running transversely beneath the abdominal wall defect in the retrorectus plane (Figure 1). A 2-0 nonabsorbable interrupted suture was placed at all four corners of the device to aid in device identification at necropsy. The device was then secured in position with transfascial interrupted \#0 prolene sutures placed at each corner and midpoint of each edge of the device. A Reverdin needle was used to pass the suture through the abdominal wall and the knots were buried in the subcutaneous tissue. In nonreinforced control animals, the transfascial sutures were still placed to eliminate any confounding variables from the immune response to the sutures and to provide landmarks for excision of the region of interest at necropsy. The abdominal wall was reapproximated with interrupted absorbable suture and the incision was closed with a double layer of running absorbable suture. Dermal glue was also applied to the incision line and transfascial suture sites to limit postoperative contamination. Antibiotics were provided intraoperatively and daily for 3 days postoperatively to all animals. Buprenorphine and nonsteroidal anti-inflammatory drugs were also provided once daily for 3 days postoperatively.

\section{Pathological evaluation}

At time points of 3 and 8 months postsurgery, the animals were humanely euthanized by intravenous injection of a pentobarbital-based solution as accepted by American Veterinary Medical Association Guidelines on Euthanasia. The incision site was photographed and a full necropsy was performed by an independent, board certified veterinary pathologist that was blinded to the study groups. The nonabsorbable transfascial anchoring sutures were identified in each animal to establish the boundaries of the area of interest. The entire defect site, extending approximately $1 \mathrm{~cm}$ beyond the identified sutures, was then explanted. The defect site was inspected for recurrence of hernia, for signs of seroma, adhesions or inflammation, and for the presence of any remaining device. An approximately 2-cm-thick piece of tissue from the cranial side of the explanted tissue, spanning the full thickness of the abdominal wall and the entire lateral width of the defect site, was removed for histological analysis. The remaining tissue was wrapped in saline-soaked gauze and fresh-frozen until time of mechanical testing. The rest of the animal was 
Table 1. Scoring rubric for histological analysis of tissue samples.

\begin{tabular}{|c|c|c|c|c|c|}
\hline Grade & 0 & 1 & 2 & 3 & 4 \\
\hline $\begin{array}{l}\text { Overall inflammation, } \\
\text { edema/seroma, } \\
\text { hemorrhage, integration } \\
\text { of implant, necrosis, } \\
\text { mineralization, fibrous } \\
\text { capsule, fibrosis, } \\
\text { microgranulomas, hernia } \\
\text { formation }\end{array}$ & Absent & Minimal & Mild & Moderate & Severe/marked \\
\hline $\begin{array}{l}\text { Polymorphonuclear cells } \\
\text { (neutrophils, eosinophils), } \\
\text { lymphocytes, plasma cells, } \\
\text { macrophages, MNGCs }\end{array}$ & Absent & $\begin{array}{l}\text { Minimal, rare, } 1-5 / \mathrm{hpf} \\
(1-2 / \mathrm{hpf} \text { for MNGCs) }\end{array}$ & $\begin{array}{l}\text { Mild, } 6-10 / \mathrm{hpf}(3-5 / \mathrm{hpf} \\
\text { for MNGCs) }\end{array}$ & $\begin{array}{l}\text { Moderate, heavy } \\
\text { infiltrate }\end{array}$ & $\begin{array}{l}\text { Severe/marked, packed } \\
\text { (sheets of MNGCs) }\end{array}$ \\
\hline Vascularization & $\begin{array}{l}\text { Absent, no } \\
\text { vascularization noted } \\
\text { within tissue }\end{array}$ & $\begin{array}{l}\text { Minimal, few scattered } \\
\text { vessels within tissue }\end{array}$ & $\begin{array}{l}\text { Mild, frequent small } \\
\text { vessels throughout tissue }\end{array}$ & $\begin{array}{l}\text { Moderate, numerous } \\
\text { small vessels throughout } \\
\text { tissue }\end{array}$ & $\begin{array}{l}\text { Marked, extensive bands of } \\
\text { vascularization throughout } \\
\text { tissue }\end{array}$ \\
\hline Fibrous capsule & $\begin{array}{l}\text { Absent, no fibrous } \\
\text { capsule surrounding } \\
\text { implant }\end{array}$ & $\begin{array}{l}\text { Minimal, narrow band of } \\
\text { fibrosis surrounds implant }\end{array}$ & $\begin{array}{l}\text { Mild, slightly thick band } \\
\text { of fibrosis surrounds } \\
\text { implant }\end{array}$ & $\begin{array}{l}\text { Moderate, thick band of } \\
\text { fibrosis surrounds implant }\end{array}$ & $\begin{array}{l}\text { Marked, extensive band of } \\
\text { fibrosis surrounds implant }\end{array}$ \\
\hline Residual implant material & $\begin{array}{l}\text { Absent (no implant } \\
\text { material present) }\end{array}$ & $\begin{array}{l}\text { Minimal, small amount of } \\
\text { implant material } \\
\text { remaining }\end{array}$ & $\begin{array}{l}\text { Mild, multiple slightly } \\
\text { thick bands of implant } \\
\text { material remaining }\end{array}$ & $\begin{array}{l}\text { Moderate, large amount } \\
\text { of implant material } \\
\text { remaining }\end{array}$ & $\begin{array}{l}\text { Marked, very extensive } \\
\text { band of implant material } \\
\text { remaining }\end{array}$ \\
\hline
\end{tabular}

then subjected to a full necropsy and any tissues deemed abnormal by the pathologist were saved for histological evaluation.

\section{Histological analysis}

Full thickness histological samples of the defect region were immersed in $10 \%$ neutral buffered formalin for 3-5 days postnecropsy, then transferred to $70 \%$ ethanol until processing. Samples were trimmed, processed and embedded in paraffin in an oversized cassette. The samples were then serially sectioned and stained with hematoxylin and eosin and Masson's Trichrome. Stained tissue sections were then provided to an independent, certified veterinary pathologist, blinded to the study groups, for semiquantitative analysis of the host response to the injury and implanted device. Table 1 shows the scoring rubric that was used for the semiquantitative analysis of the samples, with a low score corresponding to an absent or limited response and higher score corresponding to a marked response. The following parameters were specifically examined: overall inflammation, edema/seroma formation, hemorrhage, integration of the implant material, necrosis, mineralization, fibrous capsule around the implant, hernia formation, polymorphonuclear cells (neutrophils and eosinophils), lymphocytes, plasma cells, macrophages, multinucleated giant cells and vascularization. Additionally, the approximate horizontal distance between the rectus muscles was measured using an ocular micrometer and/or a ruler overlying the histology slide as an estimate of rectus diastasis.

\section{Ball burst testing}

Samples of the defect site were allowed to gradually equilibrate to room temperature prior to testing. Any excess adipose tissue remaining on the samples was manually trimmed down to the layer of the anterior fascia. The final tissue sample, from peritoneum through anterior fascia, was trimmed to a size of approximately $7.5 \times 7.5 \mathrm{~cm}$ with the defect centered in the sample. This tissue was then clamped within a Ball Burst Compression Fixture (Instron Corporation, MA, USA). A piece of 60 grit sand paper was used to prevent slippage of the tissue within the fixture. A $2.5 \mathrm{~cm}$ steel ball was then lowered into the sample at a rate of $12 \mathrm{in} / \mathrm{min}$ until the tissue ruptured. The ball burst strength values were recorded as the maximum load reading of the Instron Universal Testing System.

\section{Statistical analysis}

Statistical analyses were conducted on the mechanical data using MiniTab 16 software. Data were analyzed by a blinded technician and presented as mean \pm standard deviation. A student's t-test was utilized to compare data between the two time points for each group. A one-way analysis of variance was utilized to compare data between 
treatment groups at each time point. A p-value less than 0.05 was considered significant. Histological scoring data are presented as mean \pm standard deviation; no further statistical analysis was performed on this attribute data.

\section{Results}

\section{Surgical outcomes}

Surgeries were successfully conducted in 32 pigs without any complications. Each of the UBM-derived surgical device was placed between the rectus muscle and the posterior fascia, and all were easily secured with transfascial sutures without excessive tension (Figure 1B). All animals recovered uneventfully from surgery. A number of pigs had slight swelling/edema at the incision site and a slightly tender abdomen when palpated the day after surgery. These observations were spontaneously resolved within 3-5 days.

There were two unscheduled deaths in the study. One pig in the 8-month cohort (Group 3) developed renal failure that correlated with high serum concentrations of blood urea nitrogen (BUN), creatinine and phosphorus and was euthanized on Day 57 at the recommendation of the attending veterinarian due to suspected renal failure, which was confirmed at necropsy. This animal had elevated levels of BUN preoperatively (at the upper end of allowable range) and thus was considered to have had a predisposition to chronic nephropathy that developed into renal failure over the course of the study. While it cannot be exclusively ruled out, the causative evidence and the absence of similar reactions in the other 23 animals receiving UBM implants suggest that this outcome was not test article-related. This animal was excluded from the resulting histological and mechanical analyses as it did not reach the earliest scheduled study end point.

A second early termination was conducted on a pig in the 3-month cohort that was reinforced with a UBMderived surgical device (Group 3). This pig had gross evidence of abscess formation lateral to the midline incision site. The abscess was lanced to drain the abscess by the attending veterinarian, which resulted in the protrusion of a fibrous tissue. The animal was recommended for immediate euthanasia on Day 71 by the attending veterinarian, assuming the test article had been extruded through the abscess. Histological evaluation confirmed microscopic evidence of pyogranulomatous inflammation surrounding suture material within the abscess site and determined that the extruded tissue was in fact the fibrous capsule of the abscess and not the test article. No evidence of bacteria was found in any of the examined implant sections, nor within the retrorectus plane. The inflammation present was presumed by the pathologist to have originated from the affected suture site. The pyogranulomatous abscess extended to the presumed left side of the implant site (likely near the infected suture site), though the entire implant site was not affected by the inflammatory process and healthy fascial tissue was found in the retrorectus plane. Other devices from this same lot were also used in this study, which were all sterilized using validated radiation processes, and did not result in infections. While it cannot be absolutely confirmed, the abscess seen in this animal was presumed to be pursuant to bacterial contamination of the suture implantation site either intraoperatively or postoperatively, and not from the implanted device. This animal was also excluded from the resulting histological and mechanical analyses as it did not reach the earliest scheduled study end point. The remaining animals survived until scheduled termination without incident.

\section{Macroscopic findings}

A complete necropsy was performed on each animal by an independent board certified pathologist. All organs were within the expected weights and appearance for each of these animals (heart, liver, spleen, thymus, kidney, etc.) There was no evidence of seroma, edema or adhesions in any animal. There was also no observed herniation in any of the animals that were reinforced with a UBM-derived surgical device at either time point. Interestingly, none of the originally implanted UBM devices could be distinguished from the surrounding tissue by the 8-month time point (Figure 2A \& B). In contrast, 50\% (2/4) of the nonreinforced animals at the 8-month time point exhibited focal areas of herniation of preperitoneal adipose tissue through the incision line to the level of the anterior fascia (Figure 2C).

Examination of the implant region showed that the UBM-derived surgical devices had been replaced with fibrous connective tissue in the retrorectus plane. As a qualitative observation, the tissue planes of the abdominal wall were able to be separated with blunt dissection at both the 3-and 8-month time points. Figure 3 shows the end loop of one of the transfascial sutures that was buried in fibrous connective tissue within the retrorectus plane at 3 months. This animal had received a UBM-derived surgical device implant (Group 3), but the original device was no longer macroscopically identifiable. 


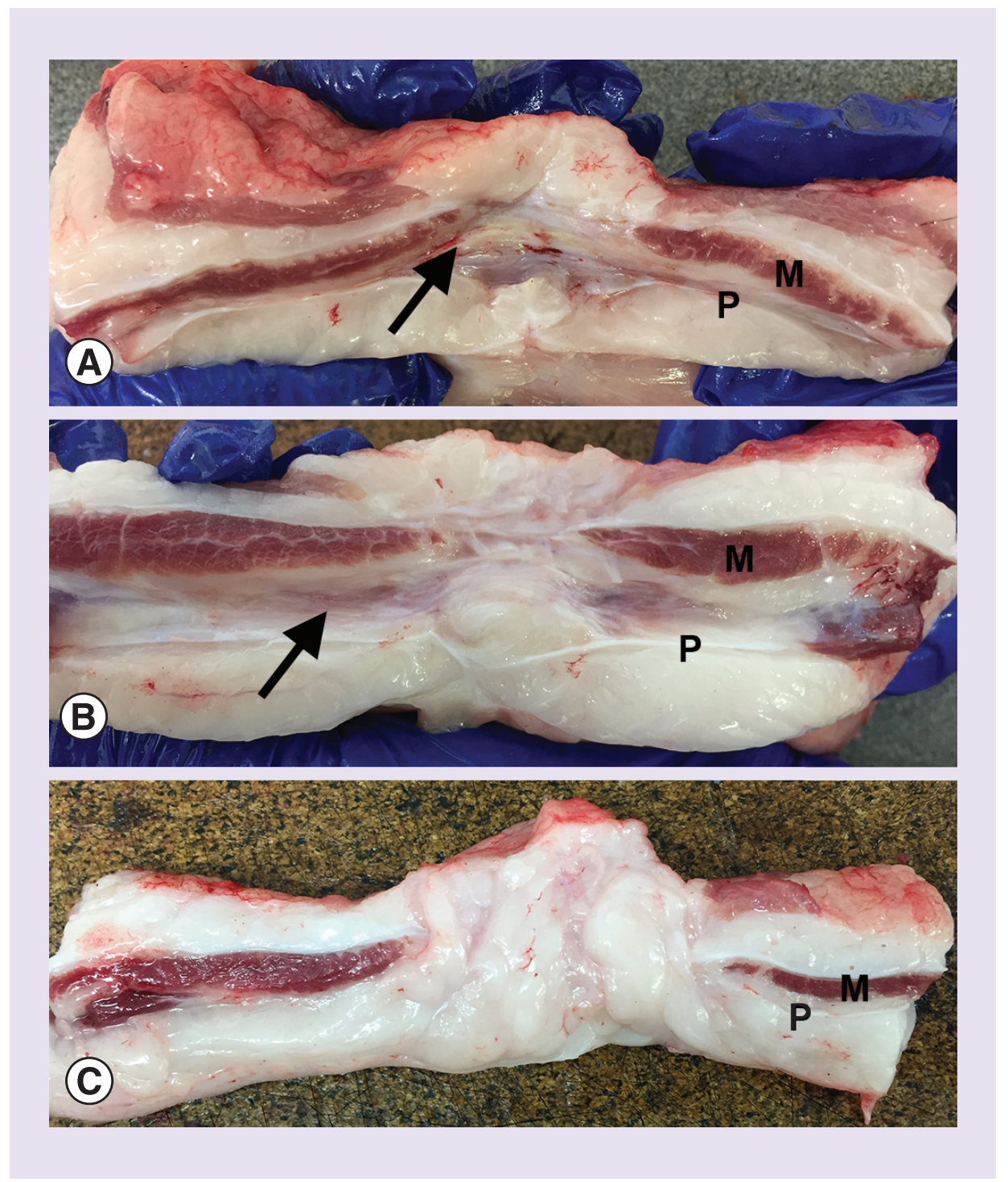

Figure 2. Necropsy results. (A) At 3 months, the majority of the UBM-derived surgical devices had been fully resorbed; only partial elements of the UBM device (arrow) could still be identified in the retrorectus plane. (B) By 8 months, all of the UBM-derived surgical devices had been completely remodeled into a loose, vascularized connective tissue (arrow indicates original placement of reinforcement device in retrorecutus plane). (C) In contrast, nonreinforced animals developed herniation of preperitoneal fat through the midline defect by 8 months. Notice the increased separation of the rectus muscles as well.

M: Rectus muscle; P: Posterior fascia; UBM: Urinary bladder matrix.

\section{Histological remodeling}

Histological analysis supported the macroscopic observations during necropsy. A semiquantitative analysis of several histologic parameters was conducted for each test group. Several key parameters are displayed in Table 2. Residual implant material was only found at the 3-month time point in one of the UBM groups (Group 4), as evidenced by small aggregates of dense collagenous tissue (Figure 4A). All other treatment groups and time points had no visible implant material remaining within the defect site, and only fibrous tissue remained (Figure 4B, D \& E). This remodeled tissue largely resembled native posterior fascia, but with a qualitatively apparent increase in overall thickness (Figure 4C). The UBM material that could be identified at the 3-month time point was characterized by an infiltration of fibroblasts and mesenchymal cells, as well as the presence of small blood vessels. There was also no evidence of fibrous encapsulation around the remaining implant material at 3 months, or in any other implant region. 
Figure 3. Remodeled urinary bladder matrix. Within 3 months, the UBM-derived surgical device had been completely remodeled. Seen here, the peritoneum and posterior fascia have been elevated to expose the loop of one of the transfascial sutures that had originally been passed through the device. At 3 months, the device was no longer identifiable and the suture was embedded in a layer of fibrous connective tissue. Dotted line denotes extent of fascial layer dissection. UBM: Urinary bladder matrix.

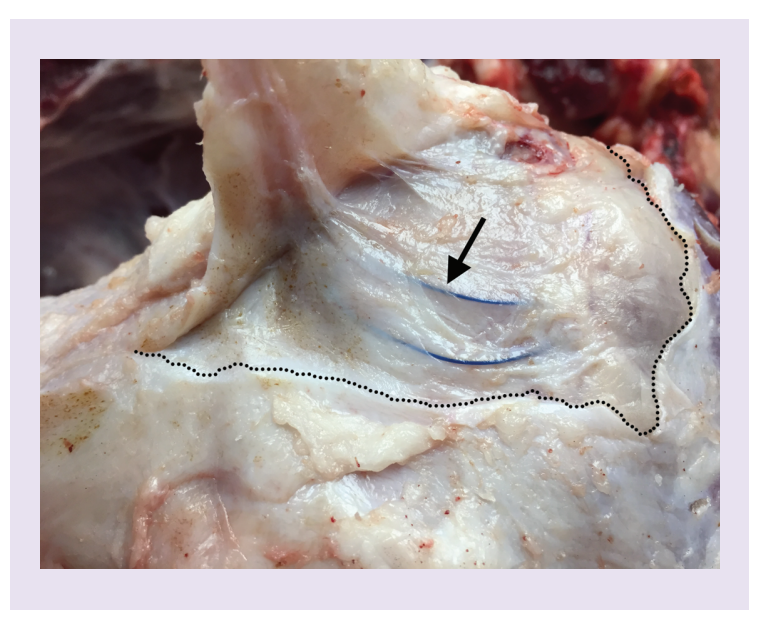

Table 2. Semiquantitative histological analysis of defect region.

\begin{tabular}{|c|c|c|c|c|c|c|c|c|}
\hline \multirow{2}{*}{$\begin{array}{l}\text { Pathologic } \\
\text { characteristic }\end{array}$} & \multicolumn{2}{|c|}{ Group 1 - Nonreinforced } & \multicolumn{2}{|c|}{ Group 2 - UBM } & \multicolumn{2}{|c|}{ Group 3 - UBM ${ }^{\dagger}$} & \multicolumn{2}{|c|}{ Group 4 - UBM } \\
\hline & 3-month & 8-month & 3-month & 8-month & 3-month & 8-month & 3-month & 8-month \\
\hline $\begin{array}{l}\text { Residual implant } \\
\text { material }\end{array}$ & $N / A$ & $\mathrm{~N} / \mathrm{A}$ & $0.0 \pm 0.0$ & $0.0 \pm 0.0$ & $0.0 \pm 0.0$ & $0.0 \pm 0.0$ & $0.8 \pm 0.6$ & $0.0 \pm 0.0$ \\
\hline $\begin{array}{l}\text { Overall } \\
\text { inflammation }\end{array}$ & $1.5 \pm 0.6$ & $2.0 \pm 0.0$ & $1.5 \pm 1.0$ & $1.3 \pm 0.5$ & $1.3 \pm 0.6$ & $1.0 \pm 0.0$ & $1.5 \pm 0.6$ & $1.0 \pm 0.0$ \\
\hline Neutrophils & $0.0 \pm 0.0$ & $0.0 \pm 0.0$ & $0.3 \pm 0.5$ & $0.0 \pm 0.0$ & $0.3 \pm 0.6$ & $0.0 \pm 0.0$ & $0.0 \pm 0.0$ & $0.0 \pm 0.0$ \\
\hline Eosinophils & $0.0 \pm 0.0$ & $0.0 \pm 0.0$ & $0.0 \pm 0.0$ & $0.0 \pm 0.0$ & $0.0 \pm 0.0$ & $0.0 \pm 0.0$ & $0.3 \pm 0.5$ & $0.0 \pm 0.0$ \\
\hline Lymphocytes & $0.5 \pm 0.6$ & $0.8 \pm 0.5$ & $0.0 \pm 0.0$ & $0.3 \pm 0.5$ & $1.3 \pm 0.6$ & $0.0 \pm 0.0$ & $1.5 \pm 0.6$ & $0.0 \pm 0.0$ \\
\hline Plasma cells & $0.0 \pm 0.0$ & $0.0 \pm 0.0$ & $0.0 \pm 0.0$ & $0.0 \pm 0.0$ & $0.0 \pm 0.0$ & $0.0 \pm 0.0$ & $0.0 \pm 0.0$ & $0.0 \pm 0.0$ \\
\hline Macrophages & $1.5 \pm 0.6$ & $2.0 \pm 0.0$ & $1.5 \pm 1.0$ & $1.3 \pm 0.5$ & $1.3 \pm 0.6$ & $1.0 \pm 0.0$ & $1.5 \pm 0.6$ & $1.0 \pm 0.0$ \\
\hline $\begin{array}{l}\text { Multinucleated } \\
\text { giant cells }\end{array}$ & $1.3 \pm 0.5$ & $2.0 \pm 0.0$ & $1.0 \pm 0.0$ & $1.3 \pm 0.5$ & $1.3 \pm 0.6$ & $0.7 \pm 0.6$ & $1.3 \pm 0.5$ & $1.0 \pm 0.0$ \\
\hline Microgranuloma & $1.0 \pm 0.8$ & $0.3 \pm 0.5$ & $0.3 \pm 0.5$ & $0.0 \pm 0.0$ & $0.7 \pm 1.2$ & $0.0 \pm 0.0$ & $0.3 \pm 0.5$ & $0.0 \pm 0.0$ \\
\hline Fibrosis & $3.0 \pm 0.8$ & $1.5 \pm 0.6$ & $2.8 \pm 1.0$ & $1.5 \pm 0.6$ & $2.7 \pm 0.6$ & $1.7 \pm 0.6$ & $3.5 \pm 0.6$ & $1.5 \pm 0.6$ \\
\hline Vascularization & $3.0 \pm 0.0$ & $2.5 \pm 0.6$ & $3.0 \pm 0.0$ & $2.5 \pm 0.6$ & $2.7 \pm 0.6$ & $3.0 \pm 0.0$ & $3.0 \pm 0.0$ & $3.0 \pm 0.0$ \\
\hline Fibrous capsule & $0.0 \pm 0.0$ & $0.0 \pm 0.0$ & $0.0 \pm 0.0$ & $0.0 \pm 0.0$ & $0.0 \pm 0.0$ & $0.0 \pm 0.0$ & $0.0 \pm 0.0$ & $0.0 \pm 0.0$ \\
\hline Hernia & $0.0 \pm 0.0$ & $1.5 \pm 1.9$ & $0.0 \pm 0.0$ & $0.0 \pm 0.0$ & $0.0 \pm 0.0$ & $0.0 \pm 0.0$ & $0.0 \pm 0.0$ & $0.0 \pm 0.0$ \\
\hline
\end{tabular}

Overall inflammation decreased for all groups across the study. By the 8-month time point, the nonreinforced animals (Group 1) had the highest average inflammation score at 2.0. The UBM-derived surgical devices showed slightly less inflammation, with a score of 1.3 for Group 2 and 1.0 for Groups 3 and 4 . The increased inflammation in the nonreinforced group also corresponded to higher levels of lymphocytes, macrophages and multinucleated giant cells compared with the animals that received reinforcement with UBM-derived surgical devices. Fibrosis within the defect site also decreased for all groups across the study. At the 8-month time point, all groups had similarly low levels of fibrosis. In contrast, vascularization of the implant region increased for all groups across the study, with UBM Groups 3 and 4 having higher scores than Group 2 and the nonreinforced Group 1. Hernia formation was noted in 50\% of the nonreinforced animals, as defined as protrusion of tissue through the repair site, compared with none in the animals that received reinforcement (Figure 4F). Similarly, the average approximate horizontal distance between the rectus muscles was greatest for the nonreinforced group at 8 months $(3.0 \pm 1.1 \mathrm{~cm})$, compared with the average distance of $0.8 \pm 0.2 \mathrm{~cm}$ for the uninjured control group. UBM reinforced groups had an average separation of $2.7 \pm 0.5 \mathrm{~cm}$ for Group 2, $2.0 \pm 1.0 \mathrm{~cm}$ for Group 3 and $2.0 \pm 0.6 \mathrm{~cm}$ for Group 4 .

There was no edema, seroma or hemorrhage noted for any group. There was also no evidence of fibrous capsule formation or abscess in the implant region in animals that received UBM-derived surgical devices. The remaining 


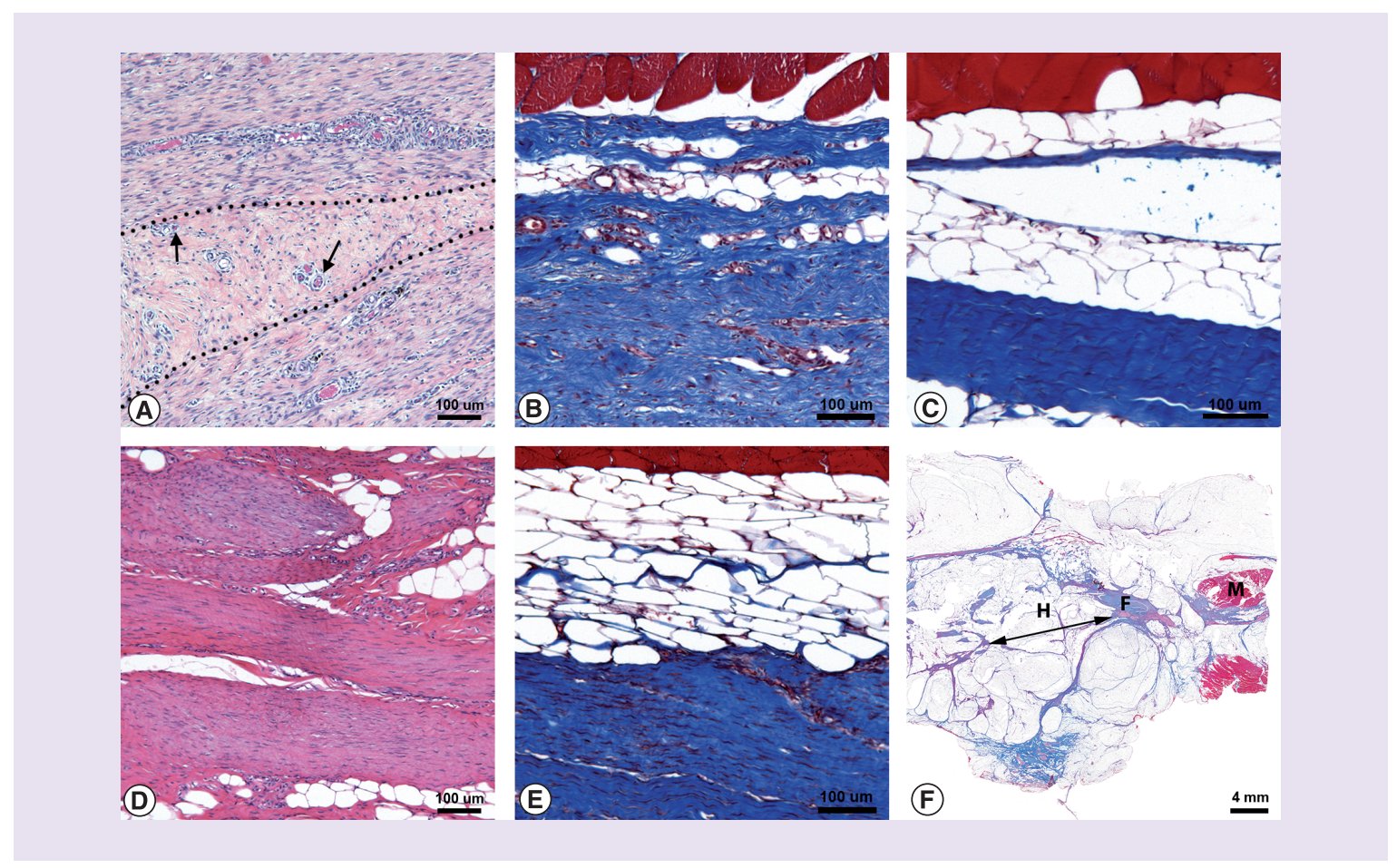

Figure 4. Histological results. (A) Hematoxylin and eosin staining of an abdominal repair reinforced with a UBM-derived surgical device (Group 4) at 3 months showed small areas of collagenous tissue where the device still remained (outlined by dotted lines). Cellular infiltration and neovascularization (arrows) could also be identified penetrating the remaining device. All other UBM-derived surgical devices were unable to be distinguished from surrounding tissue at 3 months. (B) With Masson's Trichrome staining, a thickened vascularized, collagenous tissue in the appropriate plane posterior to the rectus muscle can be seen. (C) Native posterior fascia from uninjured animals stained with Masson's Trichrome was largely similar to the tissue that had developed in response to the placement of UBM-derived surgical devices. (D \& E) By 8 months, none of the UBM-derived surgical devices could be identified and had been replaced with loose connective tissue in the retrorectus plane. There was no evidence of fibrous encapsulation. (F) Histological evaluation of tissue samples from nonreinforced animals at 8 months showed herniation (labeled with ' $\mathrm{H}$ ') of preperitoneal adipose tissue between the rectus muscles (M). Fibrosis can be seen within the defect (labeled with 'F'). UBM: Urinary bladder matrix.

histological parameters (necrosis, mineralization, polymorphonuclear cells [neutrophils, eosinophils], plasma cells) showed equivalently low values across all study groups.

\section{Mechanical strength analysis}

Samples of each implant region were also assessed for mechanical strength via ball burst testing. As seen in Figure 5, all groups increased in strength from the 3-month to the 8-month time point, with UBM Group 2 showing a statistically significant increase $(\mathrm{p}<0.05)$. At 3 months, UBM Groups 3 and 4 trended toward increased strength compared with nonreinforced animals (Group 1), but it was not a statistically significant difference $(\mathrm{p}>0.05)$. By 8 months, there was no significant difference in the strength of the surgically repaired animals (reinforced or nonreinforced) compared with the native abdominal wall of the uninjured control animals, regardless of the type of reinforcement material employed.

\section{Discussion}

This study highlights the long-term performance of three UBM-derived surgical devices in a preclinical model of ventral incisional hernia repair. Reinforcement of the primary repair successfully prevented hernia occurrence in these animals, which would have otherwise been an anticipated outcome given the $50 \%$ failure rate seen in the nonreinforced group. It also limited the extent of rectus diastasis that was seen in the nonreinforced group, as 


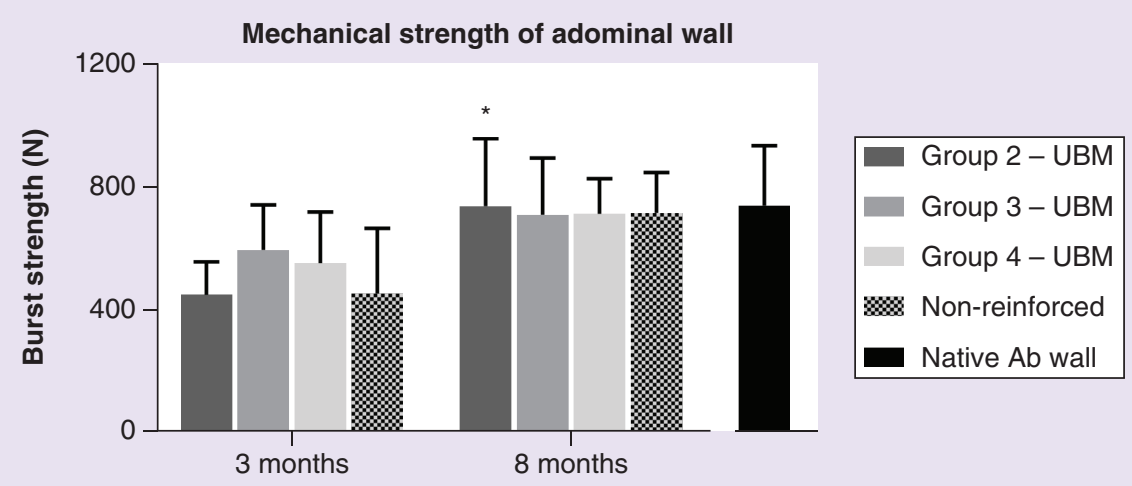

Figure 5. Mechanical strength of repaired abdominal wall. Ball burst strength of the abdominal wall (peritoneum through anterior fascia) at midline (in Newtons). The strength of the abdominal wall when reinforced with UBM-derived surgical devices (Group 2) significantly increased from 3 months to 8 months (*p $<0.05$ ). All groups had approached the mechanical strength of the uninjured native abdominal wall at 8 months. There was no statistically significant difference between groups at either time point. Note that one animal was excluded from analysis in Group 3 at both time points due to nondevice-related early termination. UBM: Urinary bladder matrix.

evidenced by the decrease in lateral distance between the rectus muscles. Rectus diastasis has previously been shown to be a significant risk factor for hernia recurrence [31].

All three UBM-derived surgical devices were associated with a positive tissue remodeling response, characterized by a muted inflammatory reaction and the absence of fibrous encapsulation. In particular, the UBM-derived surgical devices showed a trend toward lower inflammatory scores compared with the nonreinforced group. This was presumably the result of a modulation of the foreign body response to the synthetic sutures used for midline closure due to the presence of UBM. Previous studies have demonstrated that UBM materials are capable of mitigating the inflammatory response to synthetic mesh materials such as polypropylene $[21,32,33]$.

In this study, the UBM-derived surgical devices were remodeled over time into a vascularized, fibrous, siteappropriate connective tissue in the retrorectus plane. Qualitatively, this remodeled tissue largely resembled a thickened posterior fascia. Instead of being replaced with dense scar tissue, the UBM-derived surgical devices appeared to facilitate the deposition of new posterior fascia that may have contributed to the positive outcome. Furthermore, the nature of this newly developed tissue allowed easy re-entry into the retrorectus space of the abdominal wall at necropsy, contrary to the fibrotic response that would be expected had a synthetic material been similarly used [34,35]. Comparable porcine models of retrotrectus device placement have shown that synthetic materials, such as those derived from polypropylene, polytetrafluoroethylene, polyglycolic acid and poly-4-hydroxybutyrate have also demonstrated foreign body responses and encapsulation of mesh fibers in porcine models [34,36,37]. Similarly, dermal and pericardial ECM materials have been associated with dense fibrotic scar tissue and moderate encapsulation at the site of device placement [38-40]. The unique tissue remodeling response observed with UBM reinforcement could be particularly advantageous in the event that future laparotomies are required for a patient. However, additional comparative studies are warranted to confirm this observation.

Interestingly, the UBM-derived surgical devices each showed a resorption rate that was faster than what would traditionally be expected for a resorbable surgical mesh device. Polyglycolic acid-trimethylene-carbonate devices have a published resorption time of 6-7 months and poly-4-hydroxybutyrate and dermal ECM devices have been shown to have persistence in excess of 12 months [40-43]. In this study, the UBM-derived surgical devices were all completely remodeled within 8 months, with the slightly increased residence time of the Group 4 device configurations likely the result of increased initial mass and/or thickness. While specific device placement (retrorectus vs onlay vs underlay) and patient comorbidities will ultimately have the greatest influence on resorption rate of the device clinically, it was important to note the absence of herniation well after the resorption of the reinforcement device was complete in this study.

Furthermore, despite this relatively rapid resorption profile, the strength of the reinforced abdominal wall was similar to that of uninjured tissue within just 8 months. As demonstrated in the histological images, the UBM- 
derived surgical devices had been completely replaced with new tissue by 8 months. This suggested that it was the host tissue, and not the reinforcement device that was now supporting the mechanical load of the abdominal wall. Interestingly, the nonreinforced control animals also were not statistically different than the uninjured tissue as well. In this particular model, the primary mechanical integrity appeared to be from the suture knots, which were still intact at the 8-month time point. Thus, mechanical strength of the repaired abdominal wall may not be the sole indicator of a positive outcome in body wall repair, as the nonreinforced animals appeared to have adequate mechanical strength in this study but still showed a high failure rate. However, due to the relatively small sample sizes in this study, and the general well-being and healing capacity of pigs used for research, additional evaluation of this phenomenon is needed to confirm this finding.

These data are somewhat contrary to the historical view that surgical mesh devices are ranked according to their preimplantation strength and persistence. However, recent studies of crosslinked versus noncrosslinked dermal ECM materials have shown that long-term mechanical strength of the repaired tissue is not statistically different, despite the initial differences in mesh strength [38,40]. Similarly, preclinical studies of synthetic mesh have also shown a decrease in mechanical strength over time [36]. Each of these materials was associated with an encapsulation response in preclinical models, and confirmed in biopsies of patients with recurrent hernias following mesh reinforcement $[14,35]$. This suggests that preimplantation strength is not a primary predictor of a successful outcome, and that the host response to the material may play a key role in the surgical outcome, in addition to the initial device strength. The data presented here argue that perhaps an alternative approach to hernia repair is to provide appropriate mechanical support immediately postoperatively with a device that also facilitates the deposition of functional, site-appropriate tissue at the site of device placement, such that the host tissue will be capable of sustaining physiologic mechanical loading in the long-term after the host response to the material has been resolved.

The remodeling response to UBM seen in this study is supported by numerous published preclinical studies and clinical case reports. Most notably, a partial thickness abdominal wall defect model in rats was previously utilized to evaluate the host response to different commercially available surgical mesh materials [11]. In that study, the UBMderived surgical device was shown to be associated with a host response that was characterized by neovascularization, limited inflammation and encapsulation, rapid cellular infiltration and the presence of site-appropriate cell types. Furthermore, the study highlighted that this site appropriate remodeling response was associated with an increased ratio of anti-inflammatory (M2) to proinflammatory (M1) macrophages. On a larger scale, a porcine model of hiatal hernia repair was developed to evaluate the performance of a UBM-derived surgical device to reinforce a crural defect repair [44]. In that study, reinforcement with the UBM-derived surgical device led to robust tissue deposition at the defect site within 2 months and improved mechanical performance of the diaphragmatic tissue compared with a nonreinforced repair, even after device resorption. There were also no noted esophageal intrusions or adhesions to the surrounding viscera. Similarly, retrospective case reports have also shown positive clinical outcomes after reinforcement of hiatal hernia repairs with UBM-derived surgical devices. These studies reported no recurrences with follow-up out to an average of 37 months (range: 24-56 months) in one study and 11 months (range: 3-18 months) in another [29,45]. Collectively, these studies support the concept that an accelerated resorption profile of a surgical mesh, if complemented by functional, site-appropriate remodeling of the host tissue, may be ideal for successful long-term reinforcement of hernia repair.

Porcine models of ventral incisional hernia repair have been frequently employed in literature to evaluate the performance of surgical mesh devices [40,43]. However, there has yet to be developed an animal model that fully recapitulates the clinical sequelae of a hernia [46]. While preclinical data may not be fully indicative of clinical performance, studies have demonstrated similarities in the mechanical performance of human and porcine abdominal tissues [47]. The design of this porcine study allowed for the use of clinically relevant sizes of reinforcement devices $(10 \times 15 \mathrm{~cm})$, as well as clinically relevant device placement and suturing techniques. The high incidence of hernia formation observed in the nonreinforced animals also provided validation that the UBM-derived surgical devices or resulting remodeled tissue were indeed providing necessary reinforcement throughout the study. The location of device placement, across the linea alba, also ensures the defect site was under maximal mechanical loading. Compared with other preclinical models, which utilize a bilateral approach resulting in lateral device placement (akin to Spigelian hernia repair), this study placed the device along the midline [37]. This placement would be under considerably higher loading, particularly in a quadruped and thus provide a greater challenge to device performance. Admittedly, randomized clinical trials are still paramount to establish the correlation of this preclinical data to performance in a clinical setting. 


\title{
Conclusion
}

UBM-derived surgical devices showed long-term reinforcement of a ventral incisional hernia repair in this preclinical model. The devices were capable of preventing hernia recurrence throughout the study and over time were remodeled into a vascularized connective tissue that approximated the mechanical strength of the abdominal wall pre-injury. While surgical mesh materials have traditionally been evaluated by their preimplantation strength and persistence in the body, this study suggests that resorbable UBM-derived surgical devices are capable of reinforcing ventral hernia repairs by facilitating functional, site-appropriate tissue remodeling at the site of implantation.

\section{Translational perspective}

Clinical usage and published case reports utilizing UBM for various applications, particularly in the reinforcement of soft tissue repairs, have continued to grow in recent years. This study provides a detailed histopathological evaluation of the remodeling and host response to UBM-derived surgical devices in a long-term large animal model. The model developed for this study utilized clinically relevant device sizes, placement and fixation, and also incorporated advanced clinical techniques, such as the Rives-Stoppa-Wantz retromuscular repair. Due to the abundance of surgical mesh materials clinically available for use in ventral incisional hernia repair, it is important to extensively characterize the response to materials in animal models, which can be utilized to provide insight into clinical outcomes. This study demonstrated a unique host response and remodeling profile compared with other materials investigated in similar porcine models. Based on these results, future clinical research is warranted to evaluate whether these histological differences result in clinically meaningful outcomes, such as reduced recurrence rates or surgical site complications, particularly in a setting that allows for comparison across different mesh materials.

\section{Summary points}

- A porcine model of simulated ventral incisional hernia repair was utilized to evaluate urinary bladder matrix (UBM)-derived surgical devices for soft tissue reinforcement using a Rives-Stoppa-Wantz retromuscular approach.

- Reinforcement of ventral incisional hernias with UBM-derived surgical devices prevented hernia recurrence out to 8 months, compared with $50 \%$ recurrence in nonreinforced controls.

- No severe adverse events were noted as a result of the use of UBM-derived surgical devices in these animals.

- Each of the abdominal wall planes could be easily reaccessed with simple blunt dissection at necropsy in animals receiving reinforcement with UBM-derived surgical devices.

- UBM-derived surgical devices showed full integration within 8 months, and had been replaced with vascularized fibrous tissue similar to that of posterior fascia.

- The strength of the abdominal wall, whether reinforced with UBM-derived surgical devices or left as a primary repair, was similar to that of uninjured tissue at 8 months.

- UBM-derived surgical devices were remodeled into site-appropriate biomechanically functional tissue within 8 months.

\begin{abstract}
Acknowledgements
The authors would like to thank N Ehrhart and K McAbee for surgical assistance, the staff at Preclinical Research Services Inc. for in-life animal support and the histopathology staff at Alizée Pathology LLC, most notably C Smith and K Gibbs, for their assistance with the production of histology slides and data quality control and summarization, respectively.
\end{abstract}

\section{Financial \& competing interests disclosure}

This study was financially supported by ACell Inc. DA Young and TW Gilbert are employees of and own stock in ACell Inc. CEM Brathwaite and CA Ronaghan are on the speakers bureau for ACell Inc. The authors have no other relevant affiliations orfinancial involvement with any organization or entity with a financial interest in or financial conflict with the subject matter or materials discussed in the manuscript apart from those disclosed.

No writing assistance was utilized in the production of this manuscript.

\section{Ethical disclosure}

The study design and animal usage were reviewed and approved by the Institutional Animal Care and Use Committee for compliance with all applicable regulations and welfare laws and policies. All animal procedures and housing were in compliance with the 
USDA Animal Welfare Act, the Guide for the Care and Use of Laboratory Animals, the Guide for the Care and Use of Agricultural Animals in Research and Teaching, Public Health Service National Institutes of Health Office of Laboratory Animal Welfare, and the American Veterinary Medical Association Guidelines for Euthanasia.

Open access

This work is licensed under the Attribution-NonCommercial-NoDerivatives 4.0 Unported License. To view a copy of this license, visit http://creativecommons.org/licenses/by-nc-nd/4.0/

\section{References}

Papers of special note have been highlighted as: • of interest; $\bullet \bullet$ of considerable interest

1. Poulose BK, Shelton J, Phillips S et al. Epidemiology and cost of ventral hernia repair: making the case for hernia research. Hernia 16(2), 179-183 (2012).

2. Le Huu Nho R, Mege D, Ouaissi M, Sielezneff I, Sastre B. Incidence and prevention of ventral incisional hernia. J. Visc. Surg. 149(5 Suppl.), E3-E14 (2012).

3. Luijendijk RW, Hop WC, Van Den Tol MP et al. A comparison of suture repair with mesh repair for incisional hernia. N. Engl. J. Med. 343(6), 392-398 (2000).

4. Burger JW, Luijendijk RW, Hop WC, Halm JA, Verdaasdonk EG, Jeekel J. Long-term follow-up of a randomized controlled trial of suture versus mesh repair of incisional hernia. Ann. Surg. 240(4), 578-583 (2004).

5. Davila DG, Parikh N, Frelich MJ, Goldblatt MI. The increased cost of ventral hernia recurrence: a cost analysis. Hernia 20(6), 811-817 (2016).

6. Holihan JL, Alawadi Z, Martindale RG et al. Adverse events after ventral hernia repair: the vicious cycle of complications. J. Am. Coll. Surg. 221(2), 478-485 (2015).

7. Novitsky YW, Fayezizadeh M, Majumder A, Neupane R, Elliott HL, Orenstein SB. Outcomes of posterior component separation with transversus abdominis muscle release and synthetic mesh sublay reinforcement. Ann. Surg. 264(2), 226-232 (2016).

8. Ventral Hernia Working G, Breuing K, Butler CE et al. Incisional ventral hernias: review of the literature and recommendations regarding the grading and technique of repair. Surgery 148(3), 544-558 (2010).

- Provides recommendations and a review of available literature for the current techniques and materials to use for clinical hernia repair and proposes a hernia grading system based on risk factor characteristics of the patient and wound.

9. Narkhede R, Shah NM, Dalal PR, Mangukia C, Dholaria S. Postoperative mesh infection - still a concern in laparoscopic era. Indian J. Surg. 77(4), 322-326 (2015).

10. Bondre IL, Holihan JL, Askenasy EP et al. Suture, synthetic, or biologic in contaminated ventral hernia repair. J. Surg. Res. 200(2), 488-494 (2016).

11. Brown BN, Londono R, Tottey $\mathrm{S}$ et al. Macrophage phenotype as a predictor of constructive remodeling following the implantation of biologically derived surgical mesh materials. Acta Biomater. 8(3), 978-987 (2012).

-. Provides a comparison of the host response to 14 different commercially available surgical mesh devices in a rodent model of abdominal wall repair.

12. Crapo PM, Gilbert TW, Badylak SF. An overview of tissue and whole organ decellularization processes. Biomaterials 32(12), 3233-3243 (2011).

13. Reing JE, Brown BN, Daly KA et al. The effects of processing methods upon mechanical and biologic properties of porcine dermal extracellular matrix scaffolds. Biomaterials 31(33), 8626-8633 (2010).

14. Cavallo JA, Roma AA, Jasielec MS et al. Remodeling characteristics and collagen distribution in biological scaffold materials explanted from human subjects after abdominal soft tissue reconstruction: an analysis of scaffold remodeling characteristics by patient risk factors and surgical site classifications. Ann. Surg. 261(2), 405-415 (2015).

15. De Silva GS, Krpata DM, Gao Y et al. Lack of identifiable biologic behavior in a series of porcine mesh explants. Surgery 156(1), 183-189 (2014).

-. Histological evaluation of biopsies from clinical patients that had received surgical mesh devices out to 2 years postoperatively.

16. Sadtler K, Sommerfeld SD, Wolf MT et al. Proteomic composition and immunomodulatory properties of urinary bladder matrix scaffolds in homeostasis and injury. Semin. Immunol. 29, 14-23 (2017).

17. Gilbert TW, Wognum S, Joyce EM, Freytes DO, Sacks MS, Badylak SF. Collagen fiber alignment and biaxial mechanical behavior of porcine urinary bladder derived extracellular matrix. Biomaterials 29(36), 4775-4782 (2008).

18. Badylak SF, Hoppo T, Nieponice A, Gilbert TW, Davison JM, Jobe BA. Esophageal preservation in five male patients after endoscopic inner-layer circumferential resection in the setting of superficial cancer: a regenerative medicine approach with a biologic scaffold. Tissue Eng. Part A 17(11-12), 1643-1650 (2011). 
19. Sicari BM, Rubin JP, Dearth CL et al. An acellular biologic scaffold promotes skeletal muscle formation in mice and humans with volumetric muscle loss. Sci. Transl. Med. 6(234), 234 ra258 (2014).

20. Remlinger NT, Gilbert TW, Yoshida M et al. Urinary bladder matrix promotes site appropriate tissue formation following right ventricle outflow tract repair. Organogenesis 9(3), 149-160 (2013).

21. Liang R, Knight K, Barone W et al. Extracellular matrix regenerative graft attenuates the negative impact of polypropylene prolapse mesh on vagina in rhesus macaque. Am. J. Obstet. Gynecol. 216(2), 153e151-153e159 (2017).

22. Nieponice A, Mcgrath K, Qureshi I et al. An extracellular matrix scaffold for esophageal stricture prevention after circumferential EMR. Gastrointest. Endosc. 69(2), 289-296 (2009).

23. Gilbert TW, Nieponice A, Spievack AR, Holcomb J, Gilbert S, Badylak SF. Repair of the thoracic wall with an extracellular matrix scaffold in a canine model. J. Surg. Res. 147(1), 61-67 (2008).

24. Agrawal V, Brown BN, Beattie AJ, Gilbert TW, Badylak SF. Evidence of innervation following extracellular matrix scaffold-mediated remodelling of muscular tissues. J. Tissue Eng. Regen. Med. 3(8), 590-600 (2009).

25. Valerio IL, Campbell P, Sabino J, Dearth CL, Fleming M. The use of urinary bladder matrix in the treatment of trauma and combat casualty wound care. Regen. Med. 10(5), 611-622 (2015).

26. Kraemer BA, Geiger SE, Deigni OA, Watson JT. Management of open lower extremity wounds with concomitant fracture using a porcine urinary bladder matrix. Wounds 28(11), 387-394 (2016).

27. Geiger SE, Deigni OA, Watson JT, Kraemer BA. Management of open distal lower extremity wounds with exposed tendons using porcine urinary bladder matrix. Wounds 28(9), 306-316 (2016).

28. Mehta A, Afshar R, Warner DL et al. Laparoscopic rectopexy with urinary bladder xenograft reinforcement. JSLS 21(1), doi:10.4293/JSLS.2016.00106 (2017) (Epub ahead of print).

29. Sasse KC, Warner DL, Ackerman E, Brandt J. Hiatal hernia repair with novel biological graft reinforcement. JSLS 20(2), doi:10.4293/JSLS.2016.00016 (2016) (Epub ahead of print).

30. Costa A, Naranjo JD, Turner NJ et al. Mechanical strength vs. degradation of a biologically-derived surgical mesh over time in a rodent full thickness abdominal wall defect. Biomaterials 108, 81-90 (2016).

- Utilizes a radio-labeled isotope to quantify the resorption profile of urinary bladder matrix based surgical devices in a rodent model of abdominal wall repair

31. Kohler G, Luketina RR, Emmanuel K. Sutured repair of primary small umbilical and epigastric hernias: concomitant rectus diastasis is a significant risk factor for recurrence. World J. Surg. 39(1), 121-126 (2015).

32. Faulk DM, Londono R, Wolf MT et al. ECM hydrogel coating mitigates the chronic inflammatory response to polypropylene mesh. Biomaterials 35(30), 8585-8595 (2014).

33. Wolf MT, Dearth CL, Ranallo CA et al. Macrophage polarization in response to ECM coated polypropylene mesh. Biomaterials 35(25), 6838-6849 (2014).

34. Melman L, Jenkins ED, Hamilton NA et al. Histologic and biomechanical evaluation of a novel macroporous polytetrafluoroethylene knit mesh compared with lightweight and heavyweight polypropylene mesh in a porcine model of ventral incisional hernia repair. Hernia 15(4), 423-431 (2011).

35. Cavallo JA, Roma AA, Jasielec MS et al. Remodeling characteristics and collagen distribution in synthetic mesh materials explanted from human subjects after abdominal wall reconstruction: an analysis of remodeling characteristics by patient risk factors and surgical site classifications. Surg. Endosc. 28(6), 1852-1865 (2014).

36. Deeken CR, Matthews BD. Comparison of contracture, adhesion, tissue ingrowth, and histologic response characteristics of permanent and absorbable barrier meshes in a porcine model of laparoscopic ventral hernia repair. Hernia 16(1), 69-76 (2012).

37. Scott JR, Deeken CR, Martindale RG, Rosen MJ. Evaluation of a fully absorbable poly-4-hydroxybutyrate/absorbable barrier composite mesh in a porcine model of ventral hernia repair. Surg. Endosc. 30(9), 3691-3701 (2016).

38. Cavallo JA, Greco SC, Liu J, Frisella MM, Deeken CR, Matthews BD. Remodeling characteristics and biomechanical properties of a crosslinked versus a non-crosslinked porcine dermis scaffolds in a porcine model of ventral hernia repair. Hernia 19(2), 207-218 (2015).

-• Evaluated the mechanical strength and histologic response of the abdominal wall to crosslinked and noncrosslinked porcine-dermis-derived surgical meshes in a porcine model of ventral hernia.

39. Sahoo S, Baker AR, Haskins IN, Krpata DM, Rosen MJ, Derwin KA. Assessment of human acellular dermis graft in porcine models for ventral hernia repair. Tissue Eng. Part C Methods 23(11), 718-727 (2017).

40. Deeken CR, Melman L, Jenkins ED, Greco SC, Frisella MM, Matthews BD. Histologic and biomechanical evaluation of crosslinked and non-crosslinked biologic meshes in a porcine model of ventral incisional hernia repair. J. Am. Coll. Surg. 212(5), 880-888 (2011).

- Evaluated the mechanical strength and histologic response of the abdominal wall to various extracellular-matrix-derived surgical meshes (bovine pericardium, human dermis and porcine dermis) in a porcine model of ventral hernia.

41. Katz AR, Mukherjee DP, Kaganov AL, Gordon S. A new synthetic monofilament absorbable suture made from polytrimethylene carbonate. Surg. Gynecol. Obstet. 161(3), 213-222 (1985). 
42. Rosen MJ, Bauer JJ, Harmaty M et al. Multicenter, prospective, longitudinal study of the recurrence, surgical site infection, and quality of life after contaminated ventral hernia repair using biosynthetic absorbable mesh: the COBRA study. Ann. Surg. 265(1), 205-211 (2017).

43. Deeken CR, Matthews BD. Characterization of the mechanical strength, resorption properties, and histologic characteristics of a fully absorbable material (poly-4-hydroxybutyrate-PHASIX mesh) in a porcine model of hernia repair. ISRN Surg. 2013, 238067 (2013).

44. Riganti JM, Ciotola F, Amenabar A et al. Urinary bladder matrix scaffolds strengthen esophageal hiatus repair. J. Surg. Res. 204(2), 344-350 (2016).

45. Reznichenko AA. Different biologic grafts for diaphragmatic crura reinforcement during laparoscopic repair of large hiatal hernia: a 6-year single surgeon experience. J. Curr. Surg. 6(1), 6-13 (2016).

46. Vogels RRM, Kaufmann R, Van Den Hil LCL et al. Critical overview of all available animal models for abdominal wall hernia research. Hernia 21(5), 667-675 (2017).

47. Cooney GM, Lake SP, Thompson DM, Castile RM, Winter DC, Simms CK. The suture pullout characteristics of human and porcine linea alba. J. Mech. Behav. Biomed. Mater. 68, 103-114 (2017). 